\title{
Equation for Estimating Predialysis Total Body Water in Patients under Chronic Hemodialysis
}

\author{
Magdalena Castro ${ }^{1,2 *}$, Cinthya Urquidi ${ }^{3}$ and Rodrigo Orozco ${ }^{1}$ \\ ${ }^{1}$ Dialysis Unit, Clínica las Condes, Chile \\ ${ }^{2}$ Epidemiology/Research Methodology Head of the Research Unit, Academic Department, Chile
}

${ }^{3}$ Research Associate Professor in Epidemiology and Biostatistics, Universidad de los Andes, Chile

*Corresponding author: Magdalena Castro, Dialysis Unit, Epidemiology/Research Methodology Head of the Research Unit, Academic Department, Clínica las Condes, Santiago-Chile, Chile.

To Cite This Article: Magdalena C, Cinthya U, Rodrigo O. Equation for Estimating Predialysis Total Body Water in Patients under Chronic Hemodialysis. 2020 - 9(5). AJBSR.MS.ID.001436. DOI: 10.34297/AJBSR.2020.09.001436.

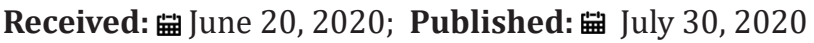

\begin{abstract}
Introduction: In Chile in 2018 there were 22,310 patients under chronic hemodialysis (CHD) (1,260/1,000,000 inhabitants), with an annual increase of 2,152 patients/year and a global mortality of $10.9 \%$. First cause of death is cardiovascular (CV) disease with $32.8 \%$. Control of total body water (TBW) helps prevent CV damage. It is necessary to know TBW to program the ultra filtration rate at the beginning of HD. Methods for estimating TBW are invasive, complex and expensive, except for multi frequency electrical Bioimpedanciometry (BCM $囚$ ).However, this device has certain limitations as the 20-40 minute preparation before HD and the restriction it has in some patients. Several equations have been proposed to estimate TBW but are difficult to calculate and neither of them are local. The aim of this study was to create a total body water equation (TBWE) to estimate TBW concordant with TBW by BCM®, easy and fast to use before each CHD session.
\end{abstract}

Method: Observational, analytical, cross-sectional, bicentric study, from January 2011 to October 2013 in Santiago, Chile. This study was approved by the institution's research ethics committee. Inclusion criteria were adults with more than 3 months of CHD, with at least 95\% HD adherence, self-sufficient and who agreed to participate in the study signing the informed consent. Patients with pacemakers, metal prostheses, an amputated limb or hospitalized were excluded. TBW previous CHD was measured with BCM ${ }^{\circledR}$ during 1st and 3rd sessions of the week; gender, age, weight, height and BMI pre CHD were also measured. TBWE elaboration consisted on a series of statistical analysis such as: Comparison of categorical variables to know if a gender-adjusted equation was needed, Pearson correlation coefficient ( $\mathrm{r}$ ), multivariate generalized linear regression modeling with significant variables, model consistency evaluation, concordance between TBWE vs. TBWBCM® using Lin's CCC and Bland Altman's Diagram. Statistical significance was established with a p-value $<0.05$. Stata12 statistical software was used.

Results: The measurements of 88 pre CHD were analyzed, in 22 male and 22 female; median age 60 years (24-77) and 56 years (22-77) respectively ( $\mathrm{p}=0.493)$; height 1.67 meters $(1.51-1.78)$ and 1.54 meters $(1.14-1.66)(p=0.001)$; previous HD weight 77.7 (55.7-132.5) and 58.7 (46.3-93.4) kgs, ( $\mathrm{p}=0.002)$; TBW pre CHD $35.2(23.3-53.6)$ and $35.2(23.3-53.6)$ liters $(\mathrm{p}=0.001)$, respectively. Final TBWE male $=-17.9+0.25 \mathrm{x}$ preHD weight $+0.19 x$ height. TBWE female $=-8.23+0.29 x$ pre CHD weight $+0.10 x$ height CCC was 84\% CI95\% 78.4-91\% p <0.001.

Conclusion: A TBWE was created for Chilean CHD patients by gender, with daily measurement clinical variables, quick and easy calculation and 84\% concordant (CI95\% 78.4-90\%) whit BCM ${ }^{\circledR}$. Its use will be feasible after validation with a larger sample.

\section{Background}

In the world and also in Chile the end-stage renal disease (ESRD) is a public health problem due to its high morbidity and mortality rates, poor prognosis and high treatment costs. Chile spends over US\$120 million per year on dialysis therapy alone. In 2018, 22,310 patients with ESRD received chronic hemodialysis (CHD).This represents a prevalence of 1,260/1,000,000 inhabitants (total 18,006,407 in habs) of which $55 \%$ are male. $38.2 \%$ of patients under CHD are diabetic and 67\%, hypertensive. Annual increase of hemodialysis patients is 2,152 /year and gross mortality rate is $11.3 \%(2,365$ patients) $[1,2]$.

The main causes of death are cardiovascular disease (32.8\%), other causes (19\%), infections $(17.3 \%)$ and cerebrovascular 
accidents (10.3\%). Primary mortality cause in patients under HD iscardiac complication, accounting for one-third of such deaths [2]. Main cardiovascular risk factor is hypertension and $68.9 \%$ of patients take at least one hypotensive treatment. Hypertension prevalence is $41.9 \%$ and $55-64.5 \%$ of hypertension in dialysis patients is associated with hydration status. Maintaining blood volume within normal ranges helps prevent development of hypertension and cardiovascular complications. In order to accomplish this, it is necessary to have an adequate blood volume monitoring and hydration control in HD patients. Controlling fluid balance and total body water (TBW) is critical to prevent cardiovascular disease [3-6].

However, it is difficult to determine a patient's hydration status by clinical signs and symptoms, such as: Edema in upper and lower extremities, jugular venous distension, dyspnea, high systolic blood pressure (SBP), increase of predialysis body weight and pulmonary edema. An objective assessment of hydration status would allow to adapt dialysis therapy on the patient's specific needs and improve accuracy in determining pre-HD extraction volumes (ultra filtration), which would result in better blood volume control. Establishing hydration status of HD patients is necessary in order to keep it within normal ranges.

A series of invasive techniques have been proposed for indirectly determining hydration status and blood volume, such as the bromide dilution method, measurements of total body potassium and deuterium dilution to estimate total body water (TBW) .The invasive nature of these techniques has restricted research. There do exist noninvasive techniques for estimating hydration status, such as dual x-ray energy absorption and measuring the inferior vena cava diameter by ultrasound [6]. However, clinical application of these methods has been limited, due to their high cost and difficulty to use them before hemodialysis [7-10].

To address this problem, many efforts and research have been carried out to find the most objective way to estimate hydration status of patient under HD. Hydration status specifically total body water (TBW) in chronic hemodialysis patients, have been and continue to be a challenge for doctors and nurses working in this area. In this context, bioelectrical impedance analysis has proven to be a reliable and feasible method to estimate patient's TBW. However, it is a procedure that needs at least 15-20 minutes time to be onset although measurement itself only takes 2 minutes. Patient must be prepared so that measurement is carried out correctly according to the manufacturer's specifications, so the estimates values are reliable.

For those who work in a chronic hemodialysis unit, time is a fundamental element that governs the beginning and end of therapy. On the other hand, patients do not want to start and end their dialysis beyond what was previously established, and these are the shift schedules already assigned for each one. For the nurses who initiate, control, and complete hemodialysis, therapy time and tolerance to the procedure are vital elements that govern the development of it. At the same time accuracy in the ultra filtration rate is necessary to eliminate enough liquid so that the patient is not left with excess or insufficient water. Consequently, it is necessary to find a simpler, faster and more reliable method to estimate predialysis total body water [13].

Hume [14] and Watson [15] built an equation to estimate total body water, but in population not in HD. Chertow M, et al. [16] built an equation for Afro-American HD patients difficult to calculate. Yanna Dou et al [17], used variables given by the BCM and studied 50 stable HD patients. Despite all the studies reported, these equations do not yet apply to daily clinical practice.

That is why, the aim of this study was to propose a simple quantitative method to estimate patient's hydration status before HD, with clinical variables measured in every dialysis session. So, it can reduce the frequency of complications and adverse events during HD procedure and improve hydration status control.

Therefore, the purpose of this study was to estimate predialysis total body water (TBW) through a simple equation comparable to the measurement of Multifrequency Electrical Bioimpedance (BCM $\left.{ }^{\circledR}\right)$, in hemodialysis patients using clinical features measurements before every dialysis.

\section{Material and Method}

Analytical cross-sectional observational study in which TBW was measured with $\mathrm{BCM} \circledast$ and the results were compared with the proposed TBW estimation equation. The study population was adult patients undergoing three-weekly HD therapy from two dialysis centers in Santiago, Chile, between January 2011 and October 2012

The study was approved by the research ethics committee of Clínica Las Condes. Informed consent of patients to participate in the study was requested, and confidentiality of information was safeguarded through patient coding and restricted access to data by a password. Inclusion criteria were adult patients undergoing three-weekly HD therapy for at least three months and who consented to participate in the study. Exclusion criteria were hospitalized patients, or with a pacemaker, or with metal prostheses or amputees.

Patients were measured with $\mathrm{BCM} 囚 20$ minutes before entering hemodialysis, and the first and third HD session of the week. To ensure measurement quality, all manufacturer's instructions were followed and only measurements that reported a quality $\geq 90 \%$ were used. A nutritionist with three years of experience measuring 
dialysis patients with $\mathrm{BCM} \AA$ was hired. Demographic variables (gender and age), usual dialysis recording clinics (predialysis weight, predialysis blood pressure), body constitution (height and BMI), total body water (TBW), overhydration and comorbidity (hypertension) were measured.

Statistical Analysis was performed with Stata 12 (Texas corp). In order to select the significant variables and build the equation, we first compared the categorical variables with $\mathrm{Chi} 2$, the continuous ones with Mann Whitney test. Pearson's (r) correlation between continuous variables and predialysis total body water (TBW) was then determined. Categorical and continuous variables that were significant (value $\mathrm{p}<0.05$ ), were included in the multivariate generalized linear Multivariate generalized linear regression model (GLM), because the linear regression did not meet the assumptions of normality. The multivariate model with the highest predictive

\begin{tabular}{|c|c|c|c|c|}
\hline \multicolumn{5}{|c|}{ Comparison of clinical characteristics by gender } \\
\hline \multirow{2}{*}{ Variables } & Female & Male & Total & \multirow{2}{*}{ p Value } \\
\hline & $n=44(50 \%)$ & $\mathrm{n}=44(50 \%)$ & $88(100 \%)$ & \\
\hline Age years & $56(22-77)$ & $60(24-77)$ & $59.5(22-77)$ & 0.493 \\
\hline Hypertension n (\%) & $9(20.4 \%)$ & $12(27.2 \%)$ & $21(23.8 \%)$ & 0.453 \\
\hline Height cm & $154.5(114-166)$ & $167(109-212)$ & $160(144-178)$ & $<0.001^{*}$ \\
\hline $\begin{array}{c}\text { Predialysis SBP } \\
\mathrm{mm} \mathrm{Hg}\end{array}$ & $135(77-207)$ & $151(109-212)$ & $145(77-212)$ & 0.121 \\
\hline $\begin{array}{c}\text { Predialysis DBP } \\
\mathrm{mm} \mathrm{Hg}\end{array}$ & $71(46-104)$ & $82.5(58-132)$ & $79.5(46-132)$ & $0.022^{*}$ \\
\hline $\mathrm{BMI} \mathrm{Kgm}^{2}$ & $24.7(21.4-36.5)$ & $28.8(23-43.8)$ & $27.3(21.4-43.8)$ & $0.003^{*}$ \\
\hline
\end{tabular}

BMI: Body Mass Index; SBP: Systolic Blood Pressure; DBP: Diastolic Blood Pressure; HBP criteria: SBP $\geq 140 \mathrm{mmHg}$ or DBP $\geq 90$ mmHg. Measurements are expressed as median (min-max), unless otherwise specified.Comparison: Categorical variables: chi2. Continuous variables: U Mann Whitney nonparametric test.Statistical significance $\mathrm{p}<0.05$

Table 2: Hydration status of the study population

\begin{tabular}{|c|c|c|c|c|}
\hline \multicolumn{5}{|c|}{ Pre-dialysis hydration status measured by BCM and stratified by gender } \\
\hline \multirow{2}{*}{ Variables } & Female & Male & Total & \multirow{2}{*}{ p Value } \\
\hline & $44(50 \%)$ & $44(50 \%)$ & $88(100 \%)$ & \\
\hline $\begin{array}{l}\text { Predialysis weight } \\
\text { Kg. }\end{array}$ & $58.7(46.3-93.4)$ & $77.7(55.7-132.5)$ & $68.7(46.3-132.5)$ & $0.002^{*}$ \\
\hline $\begin{array}{c}\text { Predialysis overhydration } \\
\text { L. }\end{array}$ & $1.35(-0.4-4.2)$ & $1.5(-1.7-6.2)$ & $1.5(-0.4-6.2)$ & 0.65 \\
\hline $\begin{array}{l}\text { Predialysis total body water } \\
\text { L. }\end{array}$ & $26.35(21.6-39.6)$ & $35.2(23.3-53.6)$ & $29.3(21.6-53.6)$ & $0.001^{*}$ \\
\hline
\end{tabular}

Median and minimum-maximum. *Significant p-value $<0.05$. Non-parametric U Mann Whitney test was used to compare variables of hydration status.

Table 3 reports the Pearson correlation (r) between predialysis TBW and predialysis Systolic blood preassure, predialysis overdydration, age, predialysis weight and height.

Table 4 shows a model that ranges from the generalized Over saturated linear one to the final model, with the significant variables. Figures 1 and 2 show the TBWEE for male and female. power was selected, using the adjusted R2 determination coefficient. The model's consistency was then evaluated with the " $n$ " sample increase simulation to 1000 through the Boostrap re-sampling system, giving the same prediction results that the original model reported. TBW estimation equation (TBWEE) had to comply with the principle of Parsimony, simple and with the greatest explanatory and predictive power. Finally, concordance between TBWEE and TBW measured with BCM was determined through the Lin's concordance correlation coefficient (CCC).

\section{Results}

Eighty-eight measurements were analyzed in 44 hemodialysis patients, $50 \%$ of whom were male. The study population characterization by gender is presented in (Table 1). Predialysis hydration status measured by BCM by gender is presented in (Table 2). 
TBWEE $_{\text {male }}=-17.976+0.251 *$ predialysis weight $+0.198 *$ height

Figure 1: Model equation for male

\section{TBWEEfemale $=-8.233+0.296^{*}$ predialysis weight $+0.109 *$ height}

Figure 2: Model equation for female

\begin{tabular}{|c|c|c|}
\hline Table 3: Pearson's $(r)$ linear correlation of total body water with the continuous variables. & P value \\
\hline Variables & TBW Pearson correlation (r) & 0.136 \\
\hline Predialysis SBP(mmHg) & 0.159 & 0.799 \\
\hline Predialysis OH(L.) & 0.027 & $0.000^{*}$ \\
\hline Heigth (m) & 0.733 & $0.000^{*}$ \\
\hline Predialysis weight(kg) & 0.855 & 0.371 \\
\hline Age(years) & -0.096 & \\
\hline TBW: Total body water; SBP: Systolic blood pressure; OH: Over hydration &
\end{tabular}

\begin{tabular}{|c|c|c|c|c|}
\hline Model variables for estimating total Predialysis body water & Estimated coefficients & Standard error & p Value & $R^{2}$ adj \\
\hline Saturated model & 0.000 & 0.012 & 0.966 & 0.764 \\
\hline Predialysis SBP & 0.142 & 0.068 & 0.038 & \\
\hline Height & 0.267 & 0.030 & 0.000 & \\
\hline Predialysis weight & -0044 & 0026 & 0089 & \\
\hline Age & -0.044 & 0.026 & 0.089 & \\
\hline Gender & 1.681 & 1.027 & 0.102 & \\
\hline Intercept & -9.132 & 10.169 & 0.369 & \\
\hline Intermediate Model & & & & 0.766 \\
\hline Height & 0.203 & 0.057 & 0.000 & \\
\hline Predialysis weight & 0.273 & 0.029 & 0.000 & \\
\hline Age & -0.037 & 0.024 & 0.126 & \\
\hline Intercept & -18.761 & 8.0618 & 0.020 & \\
\hline Final Model & & & & 0.762 \\
\hline Height & 0.209 & 0.057 & 0.000 & \\
\hline Predialysis weight & 0.271 & 0.029 & 0.000 & \\
\hline Intercept & -21.712 & 7.888 & 0.006 & \\
\hline
\end{tabular}

Table 5: Lin's Concordance correlation coefficient.

\begin{tabular}{|c|c|cc|c|}
\hline CCC & $\mathbf{n}$ & \multicolumn{2}{|c|}{$\mathbf{9 5 \%} \mathbf{~ C l}$} & P value \\
\hline 0.842 & 88 & 0.784 & 0.900 & 0.001 \\
\hline CCC $=84 \%(78.4-90 \%)$ & \\
\hline
\end{tabular}

\section{Discussion}

The study met the objective of estimating predialysis total body water (TBW) through an equation, using clinical characteristics of daily record and comparable to the measurement through the reference method, Multifrequency Electrical Bioimpedance $(B C M \cap)$, in patients with end-stage renal failure under hemodialysis therapy. The result was a predialysis total body water estimation equation (TBWEE) in adult Chilean patients with end-stage renal disease under chronic hemodialysis therapy. for each gender. Equations showed good agreement with $\mathrm{BCM} \AA$. Equation components were usual clinical recording variables in HD therapy, which have the advantage of not depending on special measurements. The equation has a simple structure which makes its calculation fast and easy, a very relevant feature when time is a fundamental element that dictates the pace and speed of action when connecting the patient to the hemodialysis (HD) procedure. All of these features make it possible for TBWEE to be used before therapy begins. This equation is intended as an input to determine more accurately and reproducibly the amount of total body water the patient has before HD. This information is intended to help the doctor and nurse schedule the ultrafiltration rate more accurately 
before the procedure begins. In addition, to further improve efficiency, an Excel with the corresponding coefficients of each equation can be set up and only the predialysis weight and height measurements need to be inserted to estimate the total body water.

In an attempt to calculate the total body water of the dialysis patient, several equations have been published. One of them is the Chertow one [15] which, according to the study of the same author and that of Seoung et al [16] 3, presented the highest correlation with bioelectrical impedance and other reference methods, in comparison with the equations of Watson and Hume $[13,14]$. As a result, we decided to find out the Chertow equation response in the patients of this study. For this purpose, the total body water (TBW) calculation was made with the TBWEE and compared with the TBW results estimated with $\mathrm{BCM} \otimes$. As a result, the Chertow equation overestimated total body water by 8 and 7.8 liters in men and women respectively. Interestingly, overestimation also occurred in the study by Seoung et al, who applied all four equations (Watson, Hume, and Chertow) in healthy Koreans and under HD, and all of them overestimated the magnitude of total body water. When Dr. Chertow was asked about the results obtained in our sample, his explanation was that the muscle mass of the Chilean patients was less than that of the population in his study, which was AfricanAmerican. This interesting finding may support the fact that it would not be appropriate to use equations built in populations other than the Chilean one. On the other hand, it is relevant to consider the complicated structure of Chertow's equation which, while using clinical variables, its structure is long enough making it not easy, nor simple and neither fast to calculate.

The result of the analysis process was the construction of two equations, one for each gender. This difference was because the coefficients of each model $(\beta 0, \beta 1$ and $\beta 2)$ were different, even though the significant variables in each equation for the prediction of total body water were the same for both male and female. Height was a significantly different variable between male and female which could explain the differences in the Beta coefficients.

When comparing the results obtained between the centers, it was not necessary to build a different equation for each one, because there were no differences between the min the variables that remained in the model. Only statistically significant differences in predialysis systolic and diastolic blood pressure were found. However, these two variables were not included in the final model because they were not significant in predicting body water. There was also no difference in weight and total predialysis body water between the two centers.

Worth mentioning is that age was not a significant characteristic when estimating TBW in both male and female, therefore, it was not included in the equation structure. When compared with the Chertow, Hume, and Watson equations, only the latter includes age in the calculation of TBW and only in female.

One of the strengths of this study is that the statistical analysis process showed that building a model to calculate total body water was feasible both for the general group as for each gender, with commonly used measurements in HD and comparable to $\mathrm{BCM} \AA$. Another one is that the equations were derived from a significant regression model with goodness-of-fit and predictive ability greater than $62 \%$ with a narrow $95 \% \mathrm{CI}$. The model proved to be robust because it was tested in a series of evaluation analyses and results showed that the variables inserted in the model did not vary (predialysis weight, height). These were evaluated in two models: linear regression and generalized linear regression. The final model was also evaluated with a re-sampling system in order to determine whether the model fit was modified if the sample " $n$ " was increased. The Bootstrap method was used for this, where a re-sampling was done 1,000 and 10,000 times. Variables, statistical significance, and adjustment remained the same. A stratified analysis by day of measurement was also carried out (measurement 1: first day of therapy, measurement 2: third day of therapy), in order to know if there could be any difference in the variables and model adjustment according to day of therapy. In this case the significant variables left in the model remained the same, predialysis weight and height. Model adjustments (R2adj) and the coefficients ( $\beta 0$, $\beta 1$ and $\beta 2$ ) had a variation of no more than $10 \%$ compared to the unadjusted model by day of measurement. Another study strength is that the final model chosen is parsimonious. This means that the simplest model with the greatest explanatory power was chosen. Finally, when evaluating concordance between body water estimated by TBWEE and body water estimated by BCM $®$ through Lin's concordance correlation coefficient (CCC) and Bland Altman, both were significant for each gender, which supports the strength and validity of the proposed equations.

One of the limitations of this study is the degree of representativeness of the target population in terms of the number of patients studied. So, the next step is to validate the equation with a larger number of subjects representing the study population in order to study TBWEE behavior and its agreement with the BCM®. We also want to include not only HD patients, but also peritoneal dialysis patients, either under continuous or intermittent ambulatory peritoneal dialysis. It is important to make clear that a sample size calculation was not performed, since the study did not pose a hypothesis to find a difference nor a certain concordance. Another study weakness is that a stratified analysis for diabetes mellitus was not performed, which will be an important point to study and determine if there is a difference in TBWEE behavior 
between patients with diabetes and those without. Perhaps this is a variable that should be included in the equation, as was done in Chertow's [15].

These study results are not intended to replace the current method used in HD to estimate patient's hydration status prior dialysis, but we seek to contribute to the quantitative determination of hydration in order to improve therapy accuracy and precision while providing useful information for safer and more effective HD.

\section{Conclusion}

A total body water estimation equation (TWBEE) was built for each gender, with clinical variables that are measured every day before dialysis. These variables are quickly and easily calculated with an accuracy and concordance with multi-frequency electrical bioimpedianciometry (BCM) of 84\% (CI95\% 78.4-90\%), which makes it feasible in daily clinical practice before each dialysis session.

\section{Conflict Disclosure}

The investigators declare no conflicts of interest. Fresenius lent the $\mathrm{BCM} \AA$ for measurements to this research.

\section{Reference}

1. Kdoqi HD (2015) Adequacy Guideline: 2015 Update. Am J Kidney Dis 66(5): 884-930.

2. Poblete H XXXVII Cuenta de hemodiálisis crónica (HDC) en Chile (al 31 de agosto de 2018) Sociedad Chilena de Nefrología.

3. Rahman M, Dixit A, Donley V, Gupta S, Hanslik T, et al. (1999) Factors associated with inadequate blood pressure control in hypertensive hemodialysis patients. Am J Kidney Dis 33(3): 498-506.

4. (2010) Team of medical affairs \& Medical information. Fresenius Medical Care. Bad Homburg. The importance of strict volume control in HD patients. Dialysis update $20(1)$.

5. Vukusich A, Fierro A, Morales J, Fantuzzi A, Vukusich C, et al. (2002) Epidemiología de la hipertensión en hemodiálisis crónica. Rev Méd Chile 130: 610-615.

6. Zazgornik J, Biesenbach G, Forstenlehner M, Stummvoll K (1997) Profile of antihypertensive drugs in hypertensive patients on renal replacement therapy (RRT). Clin Nephrol 48(6): 337-340.

7. Mesa MS (2009) Métodos para la estimación de la composición corporal II Unidad Docente Antropología Física. Facultad de Biología. Universidad Complutense de Madrid. Sociedad española de dietética y ciencias de la alimentación, 2009.

8. Agarwal R, Bouldin JM, Light RP, Garg A (2011) Inferior Vena Cava Diameter and Left Atrial Diameter Measure Volume but Not Dry Weight. Clin J Am Soc Nephrol 6(5): 1066 -1072.

9. Lee SW, Song JH, Kim GA, Lim HJ, Kim MJ (2003) Plasma brain natriuretic peptide concentration on assessment of hydration status in hemodialysis patients. Am J Kidney Dis 41(6): 1257-1266.

10. Agarwal R (2013) B-type natriuretic peptide is not a volume marker among patients on hemodialysis. Nephrol Dial Transplant 28(12): 3082 3089 .

11. Passauer J, Petrov H, Schleser A, Leicht J, Pucalka K (2010) Evaluation of clinical dry weight assessment in haemodialysis patients using bioimpedance spectroscopy: a cross-sectional study. Nephrol Dial Transplant 25(2): 545-551.

12. Wabel P, Chamney P, Moissl U, Jirka T. (2009) Importance of Whole-Body Bioimpedance Spectroscopy for the Management of Fluid Balance. Blood Purif 27(1): 75-80.

13. Moissl U, Peter W, Paul WC, Bosaeus I, Levin N, et al. (2006) Body fluid volumen determination via body composition spectroscopy in health and disease. Physiol Meas 27(9): 921-933.

14. Hume R, Weyers E (1971) Relationship between total body water and surface area in normal and obese subjects. J Clin Pathol 24(3): 234-238.

15. Chertow G, Lazarus JM, Lew NL, Ma L, Lowrie E (1999) Development of a population specific regression equation to estimate total body water in haemodialysis patients. Kidney Int 51(5): 1578-1582.

16. Seoung WL, Joon HS, Gyeong AK, Kyong JL, Moon JK (2001) Assessment of total body water from anthropometry- based equations using bioelectrical bioimpedance as reference in Korean adult control and haemodialysis subjects. Nephrol Dial Transplant 16(1): 91-97.

17. Yanna Dou, Li Liu, Xuyang Cheng, Liyun Cao, Li Zuo (2011) Comparison of bioimpedance methods for estimating total body water and intracellular water changes during hemodialysis. Nephrol Dial Transplant 26(10): 3319-3324. 